\title{
Growth factor release from a chemically modified elastomeric poly $(1,8$-octanediol-co-citrate) thin film promotes angiogenesis in vivo
}

\author{
Arun K. Sharma, ${ }^{1,2,3}$ Matthew I. Bury, ${ }^{1}$ Natalie J. Fuller, ${ }^{1}$ Dorota I. Rozkiewicz, ${ }^{3,4}$ Partha V. Hota, ${ }^{5}$ \\ David M. Kollhoff, ${ }^{6}$ Matthew J. Webber, ${ }^{7}$ Natalie Tapaskar, ${ }^{8}$ Jay W. Meisner, ${ }^{8}$ Patrick J. Lariviere, ${ }^{1}$ \\ Samantha DeStefano, ${ }^{1}$ Deli Wang, ${ }^{9}$ Guillermo A. Ameer, ${ }^{3,7,10}$ Earl Y. Cheng ${ }^{1,2,3}$ \\ ${ }^{1}$ Division of Pediatric Urology, Children's Memorial Hospital of Chicago, Chicago, Illinois 60614 \\ ${ }^{2}$ Department of Urology, Northwestern University Feinberg School of Medicine, Chicago, Illinois 60611 \\ ${ }^{3}$ Institute for BioNanotechnology in Medicine (IBNAM), Northwestern University, Chicago, Illinois 60611 \\ ${ }^{4}$ Department of Chemistry, Northwestern University, Evanston, Illinois 60208 \\ ${ }^{5}$ Department of Chemical Engineering, University of Michigan, Ann Arbor, Michigan 48109 \\ ${ }^{6}$ Loyola University Medical Center, Maywood, Illinois 60153 \\ ${ }^{7}$ Department of Biomedical Engineering, Northwestern University, Evanston, Illinois 60208 \\ ${ }^{8}$ Northwestern University, Evanston, Illinois 60208 \\ ${ }^{9}$ Children's Memorial Research Center, Chicago, Illinois 60614 \\ ${ }^{10}$ Department of Surgery, Northwestern University Feinberg School of Medicine, Chicago, Illinois 60611
}

Received 14 February 2011; revised 12 October 2011; accepted 13 October 2011

Published online 12 December 2011 in Wiley Online Library (wileyonlinelibrary.com). DOI: 10.1002/jbm.a.33306

\begin{abstract}
The ultimate success of in vivo organ formation utilizing ex vivo expanded "starter" tissues relies heavily upon the level of vascularization provided by either endogenous or artificial induction of angiogenic or vasculogenic events. To facilitate proangiogenic outcomes and promote tissue growth, an elastomeric scaffold previously shown to be instrumental in the urinary bladder regenerative process was modified to release proangiogenic growth factors. Carboxylic acid groups on poly(1,8-octanediol-co-citrate) films (POCfs) were modified with heparan sulfate creating a heparan binding POCf (HBPOCf). Release of proangiogenic growth factors vascular endothelial growth factor (VEGF), fibroblast growth factor 2 (FGF2), and insulin-like growth factor 1 (IGF-1) from HBPOCfs demonstrated an approximate threefold increase over controls during a 30-day time course in vitro. Atomic force microscopy demonstrated significant topological differ-
\end{abstract}

ences between films. Subcutaneous implantation of POCf alone, HBPOCf, POCf-VEGF, and HBPOCf-VEGF within the dorsa of nude rats yielded increased vascular growth in HBPOCf-VEGF constructs. Vessel quantification studies revealed that POCfs alone contained $41.1 \pm 4.1$ vessels $/ \mathrm{mm}^{2}$, while HBPOCf, POCf-VEGF, and HBPOCF-VEGF contained $41.7 \pm 2.6,76.3 \pm 9.4$, and $167.72 \pm 15.3$ vessels $/ \mathrm{mm}^{2}$, respectively. Presence of increased vessel growth was demonstrated by CD31 and VWF immunostaining in HBPOCfVEGF implanted areas. Data demonstrate that elastomeric POCfs can be chemically modified and possess the ability to promote angiogenesis in vivo. (c) 2011 Wiley Periodicals, Inc. J Biomed Mater Res Part A: 100A: 561-570, 2012.

Key Words: angiogenesis, elastomer, localization, growth factor, blood vessel

How to cite this article: Sharma AK, Bury MI, Fuller NJ, Rozkiewicz DI, Hota PV, Kollhoff DM, Webber MJ, Tapaskar N, Meisner JW, Lariviere PJ, DeStefano S, Wang D, Ameer GA, Cheng EY. 2012. Growth factor release from a chemically modified elastomeric poly(1,8-octanediol-co-citrate) thin film promotes angiogenesis in vivo. J Biomed Mater Res Part A 2012:100A:561-570.

\section{INTRODUCTION}

Organogenesis embodies a number of highly interdependent physiological events that must be properly coordinated to establish an anatomically correct and physiologically functional organ. Regenerative medicine-based strategies attempt to closely mimic these processes so that organ replacement therapies may eventually become a reality. Within the realm of urinary bladder regenerative medicine, many of these requirements have gone unfulfilled. Instances in which patients are undergoing corrective surgical procedures to repair urinary bladder defects such as those found in spina bifida associated neurogenic bladder often demonstrate short- and long-term side effects. ${ }^{1}$ Although attempts have been made to create a physiologically functional urinary bladder, only partial regeneration in the forms of urinary bladder constituents such as muscle and urothelial tissues has been achieved. ${ }^{2}$ This is also often accompanied by a marginal vascular supply within the core of the grafted 
area that may contribute to increased scar formation, thus decreasing bladder contractile/expansion potential. ${ }^{3,4}$ The angiogenic process in implanted bladder tissue that has been used as a "patch" during bladder augmentation procedures is typically limited in its ability to create complex interconnected vascular networks and is characterized by poor vessel integrity unless omentum is applied to the augmented tissue. ${ }^{5}$ Furthermore, a robust vasculogenic process involved with complete bladder replacement procedures has yet to be demonstrated. Hence, a method in which to enhance angiogenic events would be highly desirable in a bladder regenerative milieu.

Several proangiogenic growth factors contain internal amino acid sequences that allow for coupling to motifs found in naturally occurring molecules. The Cardin-Weintraub consensus amino acid sequence and other amino acid sequences possess heparin binding domains found in growth factors such as vascular endothelial growth factor (VEGF) and members of the fibroblast growth factor (FGF) family that favor efficient binding to molecules of heparin..$^{6-8}$ This in turn provides a potential vehicle of proangiogenic growth factor delivery for in vivo applications. The extended release of growth factors in localized areas of implanted scaffold/cell composites would provide an invaluable pillar for further tissue growth as localized growth factor delivery to enhance vascular development, and maturation has been proven to be advantageous in a number of in vivo settings., 90

Scaffold choice is critical in the urinary bladder regenerative process, since it should be able to mimic and withstand repeated cycles of mechanical strain without permanent deformation or loss of tensile strength. Dahms et al. describe various mechanical testing parameters of the human bladder and reveal that it possesses an elastic modulus of $\sim 250$ $\mathrm{kPa}^{11}$ The family of poly(diol citrates) has demonstrated its abilities to function within tissues with high elastomeric requirements. ${ }^{12,13}$ We have recently demonstrated the utility of the elastomeric scaffold poly(1,8-octanediol-co-citrate) (POC) in the context of urinary bladder regeneration. ${ }^{14}$ This study demonstrated the feasibility of mesenchymal stem cell (MSC) seeded POC scaffolds and their ability to regenerate the muscle component of the bladder wall in an established bladder augmentation model. The contractile/expansive mechanical forces endured by the POC/MSC composite helped propel the regenerative process as it has been demonstrated that scaffold mechanical stimulation promotes tissue regeneration. $^{15}$ To ultimately facilitate the regenerative processes involved with urinary bladder regeneration, we have created a chemically modified heparan binding POC film (HBPOCf) with the potential to release proangiogenic growth factors in an in vivo environment and promote localized vascular growth as a proof of concept study.

\section{MATERIALS AND METHODS}

HBPOCf synthesis and growth factor loading

POCfs were prepared as previously described. ${ }^{12-14}$ HBPOCfs were created by activating the carboxylic acid groups found on POCfs in 10-mL MES buffer [2-(N-morpholino)ethanesulfonic acid] $\mathrm{pH}$ 6.0, containing 0.15-g 1-ethyl-3-(3-dimethyla- minopropyl) carbodiimide hydrochloride (EDC) and 0.09-g $\mathrm{N}$-Hydroxysuccinimide (NHS) for $2 \mathrm{~h}$ at room temperature with agitation. Films were subsequently rinsed three times in water and incubated in 10-mL MES buffer $\mathrm{pH}$ 6.0, containing 75-mg PEG-diamine (MW 2000) for $12 \mathrm{~h}$ at room temperature with agitation. Films were then rinsed three times with water. Heparan sulfate (Celsus Laboratories, Cincinnati, $\mathrm{OH}$ ) was activated by adding 0.4-g EDC and 0.097-g NHS to a $1 \%$ solution of heparan sulfate in $50 \mathrm{~mL}$ of MES buffer $\mathrm{pH} 6.0$ for $1 \mathrm{~h}$ at room temperature. POCfs were incubated in the activated heparan sulfate solution for $4 \mathrm{~h}$ at room temperature under agitation. Films were rinsed three times in water and stored in Dulbecco's Phosphate Buffered Saline (DPBS, BioWhittaker, Walkersville, MD). A toluidine blue assay was performed to determine the amount of heparan bound to HBPOCfs. A $0.5 \mathrm{~mL}$ of toluidine blue solution $(0.005 \%$ toluidine blue in $0.01 \mathrm{M} \mathrm{HCl}$ with $0.2 \% \mathrm{NaCl}$ ) was added to a $15-\mathrm{mL}$ tube. Subsequently, $1 \mathrm{~mL}$ of $0.2 \% \mathrm{NaCl}$ and the HBPOCfs were added to the tube and vortexed for $30 \mathrm{~s}$. A $1 \mathrm{~mL}$ of hexane was added to the mixture followed by another $30 \mathrm{~s}$ of vortexing. 100-200 $\mu \mathrm{L}$ samples of the aqueous layer was removed and placed in a 96-well plate and read at 631-nm absorbance using a Spectramax M5 Plate Reader (Molecular Devices, Sunnyvale, CA).

\section{Mechanical testing of POCfs}

Tensile mechanical tests were conducted using an Instron 5544 mechanical tester (Instron, Grove City, PA) equipped with a $500 \mathrm{~N}$ load cell. Dog bone shaped samples of POCfs $(5.0 \mathrm{~cm} \times 1.0 \mathrm{~cm})$ were pulled at a rate of $500 \mathrm{~mm} / \mathrm{min}$, and the Young's modulus of the POCfs was determined by a regression of the linear portion (slope) of the tensile stress/ strain data calculated using the sum of least squares method in Excel 2007 (Microsoft Corporation, Redmond, WA). ${ }^{14}$ Data are represented as means $\pm 95 \%$ confidence intervals.

\section{Atomic force microscopy}

Tapping mode atomic force microscopy (TM-AFM) was performed with a Dimension ICON P scanning probe microscope (Veeco, Plainview, NY). The probe was composed of silicon with an aluminum reflex coating, with a spring constant of $42 \mathrm{~N} / \mathrm{m}$, a resonant frequency of 300 (200-400) $\mathrm{kHz}$, and a tip radius of $9 \pm 2 \mathrm{~nm}$ (Asylum Research, Santa Barbara, CA). Image analysis was performed with NanoScope Analysis version 1.2 software. After scanning, the images were subjected to first-order plane fitting and flattening to remove curvature and slope. The average surface roughness $\left(R_{\mathrm{a}}\right)$ was calculated on a $2.56 \mu \mathrm{m}^{2}$ area $(1.6 \mu \mathrm{m}$ $\times 1.6 \mu \mathrm{m})$ of each sample and was obtained from a minimum of three separate images acquired from different regions of the POCf, POCf-VEGF, or HBPOCf-VEGF samples. All analyses were conducted on dry, cleaned samples at room temperature in an atmosphere with $30 \%$ relative humidity. $^{16}$ 


\section{Growth factor loading and release quantification from} POCfs

The human growth factors vascular growth factor (VEGF), fibroblast growth factor 2 (FGF2), and insulin-like growth factor 1 (IGF-1) (100 ng/mL POCf each in vitro or $300 \mathrm{ng} /$ scaffold in vivo; R and D Systems, Minneapolis, MN) were individually mixed into a DPBS solution containing $0.1 \%$ bovine serum albumin [BSA (release media), Sigma Aldrich, St. Louis, MO] by manual pipetting. About $500 \mu \mathrm{L}$ of this solution was added to half-moon cutouts of POCfs, HBPOCfs, or HBPOCfs-VEGF and placed in individual 1-mL Eppendorf tubes for $3 \mathrm{~h}$ at room temperature with gentle agitation. Half-moon cutouts were $5 \mathrm{~mm}$ in diameter and $2.5 \mathrm{~mm}$ in width. The thicknesses of the films were as follows: POCf ( $n$ = 10) $0.24 \pm 0.05 \mathrm{~mm}(\mathrm{SD}) ; \operatorname{HBPOCf}(n=10) 0.25 \pm 0.05$ $\mathrm{mm}$ (SD); and HBPOCf-VEGF $(n=10) 0.24 \pm 0.06 \mathrm{~mm}$ (SD). Subsequently, the films were removed and washed three times with $200 \mu \mathrm{L}$ of DPBS and placed in a 48-well plate (Corning Life Sciences, Lowell, MA). Samples were placed in a $37^{\circ} \mathrm{C}$ incubator with $5 \% \mathrm{CO}_{2}$ in air and collected on days 1-6 and then on days $9,11,13,15,20,25$, and 30 by removing $200 \mu \mathrm{L}$ of conditioned release media and replacing with $200 \mu \mathrm{L}$ of fresh release media. Samples then underwent growth factor quantification with the appropriate enzyme-linked immunosorbent assay (ELISA) Assay ( $\mathrm{R}$ and D Systems) for the detection of the aforementioned human growth factors, as previously described. ${ }^{17-19}$ The supernatants were briefly centrifuged and placed in their respective ELISAs and assayed according to manufacturer's instructions. Samples were run in triplicate for each growth factor at each time point. Absorbance was read using the Spectramax M5 Plate Reader at wavelengths of 450 and 540 $\mathrm{nm}$. Data represented as amount of growth factor (pg) released relative to the total surface area of the scaffold $\left(22.58 \mathrm{~mm}^{2}\right)$.

\section{Subcutaneous implantation of growth factor containing POCfs}

Adult athymic female nude rats (NCI Animal Production Program, Frederick, MD) weighing $\sim 200-250$ g underwent subcutaneous scaffold implantation as previously described. ${ }^{20}$ Animals were anesthetized with $60-\mathrm{mg} / \mathrm{kg}$ ketamine and 5$\mathrm{mg} / \mathrm{kg}$ xylazine intraperitoneally and given an analgesic injection of Buprenex (1 mg/kg) administered subcutaneously to decrease any pain during and after surgery. Three $0.5-\mathrm{cm}$ incisions were made on the right, dorsal aspect of the animal within the thoracic and lumbar regions of the back. The quadrant of dorsum used for each scaffold implantation was rotated for each successive animal, to control for any effects of the implant site. The incisions were widened to accommodate POCfs or HBPOCfs (1.0-cm diameter), which were implanted into the subcutaneous layer of the dorsum of the animal. Incisions were closed with 9-mm autoclips, and a topical triple antibiotic ointment was applied to incision areas. This procedure was repeated on the contralateral side of the animal for a total of six implanted scaffolds per animal ( $n=3-5$ total animals). Animals were sacrificed at 4 weeks post-implantation. Full thickness tissue sections from the skin to the muscle were dissected and prepared for histological analyses. All pre- and post-animal procedures were performed in accordance with guidelines set forth and approved by the Children's Memorial Hospital Institutional Animal Care and Use Committee (IACUC). NIH guidelines for the care and use of laboratory animals (NIH Publication \#85-23 Rev. 1985) have been observed.

\section{Masson's Trichrome staining of implanted HBPOCfs}

Subcutaneous tissue specimens were isolated immediately following euthanasia and were fixed in 10\% buffered formalin phosphate (Fisher Scientific, Pittsburgh, PA) and dehydrated through a series of graded ethanol exchanges then embedded in paraffin. Embedded tissues were sectioned onto glass slides at a thickness of 5-15 $\mu \mathrm{m}$ using a RM2125 RT Microtome (Leica, Nannockburn, IL) and subjected to Masson's Trichrome (Sigma-Aldrich) staining as previously described. ${ }^{14}$ Following de-paraffinization, slides were placed in Bouin's solution for $15 \mathrm{~min}$, then rinsed under running tap water, and followed by Hematoxylin and Scarlet-Acid Fuchsin staining for 5 min sequentially. Following a rinse with deionized water, slides were subjected to a mixture of PTA/PMA, followed by Aniline Blue solution, and a 1\% acetic acid wash. Finally, slides were placed in 95\%-100\% ethanol and rinsed in xylene. After drying, a coverslip was placed over the tissue and secured with 3-4 drops of Permaslip (Alban Scientific, St Louis, MO).

\section{Immunofluorescent characterization of subcutaneously implanted tissues}

Slides containing sectioned tissue samples were subjected to antigen retrieval consisting of $15 \mathrm{~min}$ of boiling in citrate buffer $(0.01 \mathrm{M}$ citrate solution, $\mathrm{pH} 6.0$ with $0.05 \%$ Tween$20)$ and then cooled to room temperature. Staining consisted of a blocking step for $15 \mathrm{~min}$ in BSA ( $5 \mathrm{mg} / \mathrm{mL}$ ) followed by a 40-min incubation at room temperature with the primary antibody. After washing with DPBS, slides were incubated for 30 min with a secondary antibody and eventually rinsed with DPBS and air dried. Slides were mounted with Vectashield (Vector Laboratories, Burlingame, CA). The primary antibodies used in this study were directed against epitopes of known endothelial cell origin including PECAM (CD31) and von Willebrand Factor (vWF; Santa Cruz Biotech, Santa Cruz, CA). ${ }^{21}$ Antibodies were either directly conjugated to fluorescein isothiocyanate (FITC) or used in conjunction with a FITC conjugated secondary antibody (Molecular Probes, Carlsbad, CA) following established protocols. ${ }^{13}$ Primary antibodies were used at dilutions ranging from $1: 100$ to $1: 250$, and the secondary antibody was used at a 1:400 dilution. Samples were additionally stained with 4',6-diamidino-2-phenylindole (DAPI) to identify cell nuclei.

\section{In vivo vessel density quantification}

Trichrome images were characterized using a Nikon Eclipse 50i Microscope (Nikon, Melville, NY) and Spot Advanced Imaging Software (Diagnostic Instruments, Sterling Heights, MI). Sample images (1600 pixels $\times 2000$ pixels, bit depth 24) were opened with Adobe Photoshop CS3 (Adobe Systems, San 
Jose, CA). Images $(n=12)$ were taken from each implant and extended across the entire length of the implant with a total imaged area of $0.79 \mathrm{~mm}^{2}$. The number of vessels was quantified using the pen tool by way of the shape tools setting. Individual vessels were selected manually and subsequently quantified using the image histogram tool to acquire pixel density for each vessel. Data represented as mean number of vessels per $\mathrm{mm}^{2}, \pm 95 \%$ confidence intervals.

\section{Statistical analyses}

Growth factor release data are represented as raw data points with fitted lines and 95\% predictive intervals. Polynomial regression in a linear mixed model was used to fit data values over time. Comparisons between different constructs were considered at each time point. $p$-values were adjusted with the Tukey-Kramer method for multiple comparisons; adjusted $p$-values of $<0.05$ were considered significant. Implant data are shown as mean $\pm 95 \%$ confidence intervals. Differences between control (nonmanipulated animals), POCf, HBPOCf, POCf-VEGF, and HBPOCf-VEGF implant groups, with respect to the number of vessels, were analyzed using a linear mixed model, with the Tukey-Kramer adjustment for multiple comparisons; adjusted $p$-values of $<0.05$ were considered significant. Analyses were performed with SAS 9.2 software (SAS Institute, Cary, NC). Release data plots were generated using R Software (The R Foundation for Statistical Computing, www.r-project.org).

\section{RESULTS}

\section{Pocf mechanical characteristics}

Synthesized POCfs, HBPOCfs, and HBPOCfs-VEGF were evaluated for their stiffness by undergoing tensile mechanical stress evaluation (Fig. 1). The Young's modulus for standard POCfs was calculated to be $0.925 \pm 0.173 \mathrm{MPa}(n=12)$ in comparison with $0.806 \pm 0.010 \mathrm{MPa}(n=12)$ for the HBPOCfs and $0.752 \pm 0.097 \mathrm{MPa}(n=13)$ for the HBPOCfs-VEGF. The mechanical data suggest that modification of POC films with either heparan sulfate and/or VEGF does not significantly alter the elasticity of the film. Further mechanical testing data demonstrated an elongation at break (Eb) of 304.61\% $\pm 1.77 \%, 306.52 \% \pm 2.36 \%$, and $305.91 \% \pm 1.67 \%$ for POCf, HBPOCf, and HBPOCf-VEGF, respectively. The elongation break is defined as the percentage of the original length of the elastomer at breaking point. All groups of POCfs retained their elastic potential and pliability when manipulated. Furthermore, permanent deformation after stretching was not observed.

\section{Atomic force microscopy measurements}

Surface morphology of POCfs, HBPOCfs, and HBPOCfs-VEGF was investigated using TM-AFM in air. All groups of POCfs displayed textured surface morphology with a typical feature size of 50-300 nm. Figure 2 contains representative AFM height images and cross-sectional analyses of POCfs, HBPOCfs, and HBPOCfs-VEGF. The most uniform surface asperity coverage was found in HBPOCf-VEGF, where the average asperity size was $130 \pm 20 \mathrm{~nm}$. Average surface roughness $\left(R_{\mathrm{a}}\right)$ is the most commonly used roughness
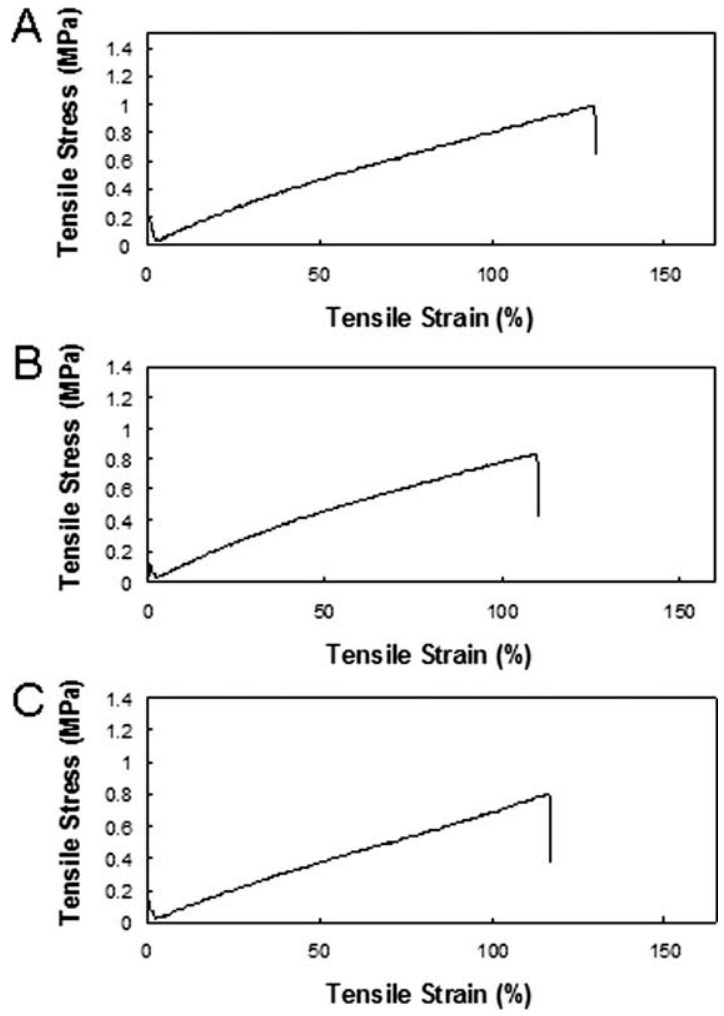

FIGURE 1. Mechanical testing data of POCfs. The modulus of each POCf was determined from the derivation of the Young's modulus (YM). (A) POCfs demonstrated a YM of $0.925 \pm 0.173 \mathrm{MPa}$. (B) HBPOCfs possessed a YM of $0.806 \pm 0.010 \mathrm{MPa}$. (C) HBPOCfs-VEGF maintained a YM of $0.752 \pm 0.010 \mathrm{MPa}$.

measurement. ${ }^{22}$ This represents the arithmetic average of the absolute values of the surface height deviations measured from the mean plane and is expressed as

$$
R_{\mathrm{a}}=\frac{1}{N} \sum_{j=1}^{N}\left|Z_{j}\right|
$$

where $N$ is the number of points, $Z$ is the difference between height and mean plane in the image, and $Z_{j}$ is the current $Z$ value. The $R_{\mathrm{a}}$ was calculated as $4.8 \pm 0.3 \mathrm{~nm}$ for POCf, $5.5 \pm 0.7 \mathrm{~nm}$ for HBPOCf, and $8.6 \pm 2.0 \mathrm{~nm}$ for HBPOCf-VEGF. The surface morphology of POCf and POCfVEGF was comparable with close $R_{\mathrm{a}}$ values. HBPOCf-VEGF showed the most distinct surface characteristics with the highest $R_{\mathrm{a}}$ values as the surface appears to have the most textured morphology (Fig. 2).

\section{Heparan sulfate binding and in vitro growth factor release quantification}

Quantification of heparan sulfate binding to POCfs resulted in an average of $6.03 \pm 1.77 \mu \mathrm{g} / \mathrm{mL}$ of heparan bound to POCfs. POCf and HBPOCf constructs were assessed with regard to growth factor loading. HBPOCf constructs showed threefold to fivefold higher levels (VEGF and IGF-1 $\sim 3 \times$ greater; FGF2 $\sim 5 \times$ greater). Loading levels were greatest for IGF-1 (POCf $34.1 \pm 9.5$; HBPOCf $116.7 \pm 3.7), \mathrm{pg}$ of $\mathrm{GF} / \mathrm{mm}^{2}$ POC for all 

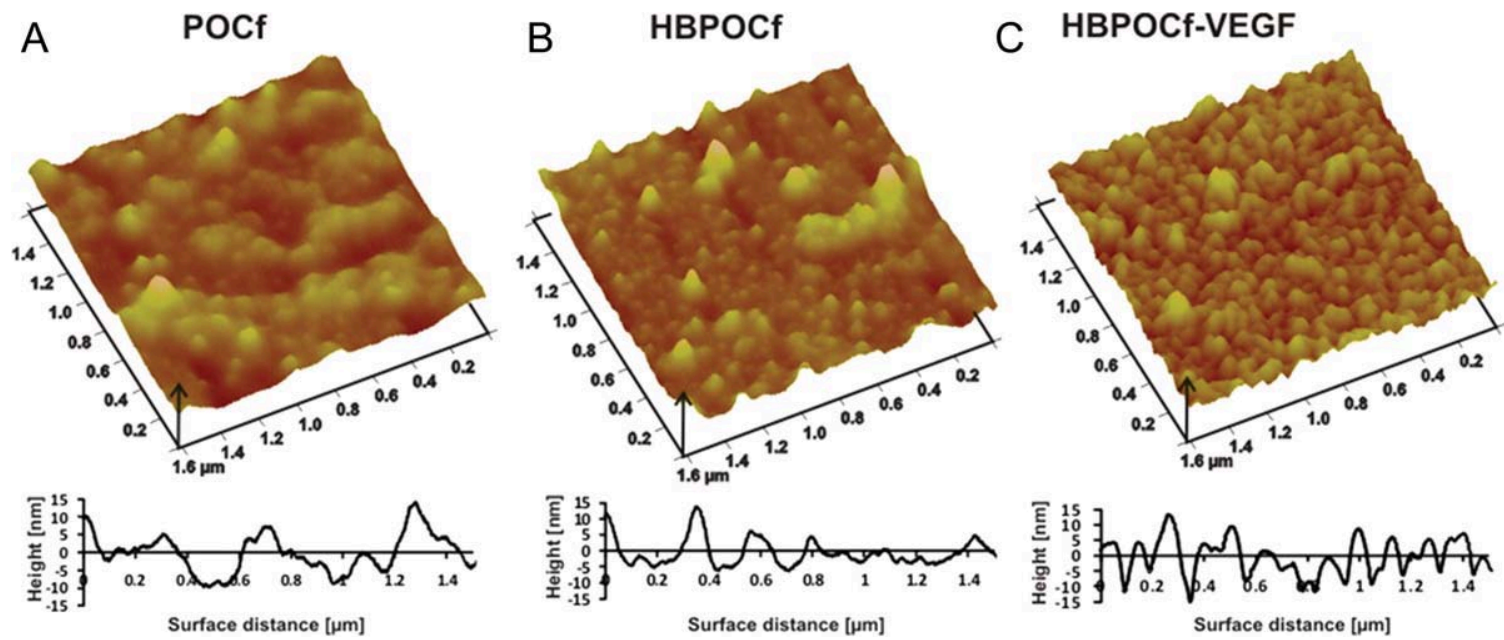

FIGURE 2. Atomic force microscopy measurements. TM-AFM three-dimensional height images of surface topography of (A) POCfs, (B) HBPOCfs, and (C) HBPOCfs-VEGF, and their cross-sectional analyses. Data indicate that average roughness $\left(R_{\mathrm{a}}\right)$ was greater in HBPOCfs-VEGF compared with controls. [Color figure can be viewed in the online issue, which is available at wileyonlinelibrary.com.]

conditions, at approximately twice the levels of VEGF (POCf $17.3 \pm 0.4$; HBPOCf $53.8 \pm 5.3)$ and six and four times the amount of FGF2 (POCf $5.3 \pm 2.2$; HBPOCf $28.2 \pm 5.0$ ). In terms of the GF available for loading, these quantities represent $1.05 \% \pm 0.30 \%$ and $3.50 \% \pm 0.14 \%$ (IGF-1; POCf, HBPOCf), $0.41 \% \pm 0.02 \%$ and $1.28 \% \pm 0.11 \%$ (VEGF; POCf, HBPOCf), and $0.14 \% \pm 0.08 \%$ and $0.75 \% \pm 0.14 \%$ (FGF2; POCf, HBPOCf), respectively. Release of VEGF, IGF-1, and FGF2 from POCf and HBPOCf constructs was monitored over a 30-day period. POCf and HBPOCf constructs were compared for each growth factor tested. In each group comparison, HBPOCf constructs released significantly greater levels of growth factor at all time points after day $1(p<0.05$ POCfFGF2 vs. HBPOCf-FGF2 at day $2 ; p<0.0001$, all other comparisons). At 30 days, IGF-1 release amounts were $87.0 \pm$ 3.1 HBPOCf versus $25.0 \pm 1.7$ POCf $\left(\mathrm{pg}\right.$ of $\mathrm{GF} / \mathrm{mm}^{2}$ POC for all conditions) [Fig. 3(A)], while VEGF release amounts were $12.9 \pm 1.8$ HBPOCf versus $4.7 \pm 0.9$ POCf [Fig. 3(B)]. Finally, FGF2 release amounts were $4.8 \pm 0.6$ HBPOCf versus $1.3 \pm$ 0.1 POCf [Fig. 3(C)]. Data are represented here as mean \pm 95\% confidence intervals. Growth factor conjugated HBPOCfs demonstrated an initial release of $\geq 20 \%$ of the total at day 1 (range $21 \%-25 \%$ ), increasing to $\geq 50 \%$ at day 4 and $\geq 75 \%$ by day 9 . Release was slightly accelerated for POCf-VEGF and POCf-IGF1, with day 1 values at $28 \%-30 \%$ total release, increasing to $\geq 50 \%$ at day 3 and $\geq 75 \%$ by day 6 . The POCfFGF2 construct followed a slower course, beginning with a lower day 1 value of $10 \%$ total release and reaching $\geq 50 \%$ (D5) and $\geq 75 \%$ (D11). Construct comparison trends (POCf vs. HBPOCf; IGF-1 vs. VEGF vs. FGF2) for release data were consistent with trends observed in loading data [Fig. 3(D)].

\section{Histological evaluation of subcutaneously implanted POCfs}

As implanted films were removed from rat backsides 4 weeks post-implantation, it was observed that the films had integrated completely into the local environment. Full thickness subcutaneous samples (consisting of skin to the muscle base) were removed and evaluated. Upon extraction of tissue/POCf composites, it was immediately obvious that significant differences in vascularity were apparent with regards to each POCf type by gross examination. Figure 4(A,E) depicts gross images of nonmanipulated POCfs, and they appear to have a lack of vascular distribution at the macroscopic level. Figure 4(B,F) depicts HBPOCf implants that demonstrate very low levels of vascularity. Figure $4(\mathrm{C}, \mathrm{G})$ shows POCf-VEGF stimulated tissue and there appears to be low levels of vascular growth as indicated by the black arrows. In contrast, Figure $4(\mathrm{D}, \mathrm{H})$ shows the HBPOCf-VEGF construct demonstrate robust vascular growth, again depicted by the black arrows, in what appears to be a localized area. Data in this image are representative of two animals, but the levels of vascular growth were consistent in all animals. Histological analyses in the form of Trichrome staining of the aforementioned constructs appear to corroborate gross findings as represented in Figure 5. Figure 5(A,F) shows tissue from a control animal that did not receive any scaffold implantation (denoted as Control in figures). Figure $5(B, G)$ contains tissue with the POCf and shows very little evidence of vascularization and is similar to untreated control samples. HBPOCf [Fig. 5(C,H)] shows sparse vessel formation. POCf-VEGF tissue containing constructs [Fig. 5(D,I)] shows slightly higher levels of vessel growth with apparent sporadic distribution. Vessels generally appear larger in diameter than POC alone samples and retain small quantities of red blood cells within the lumen of the vessel. The HBPOCf-VEGF constructs seen in Figure 5(E,J) show the greatest difference in vessel number and size. Findings were consistent throughout all animals. Figures represent dorsal tissue taken from two separate animals. Green arrows indicate blood vessels $(400 \times$ mag; scale bar $=50 \mu \mathrm{m}$ ).

\section{Immunofluorescent staining of in vivo derived POCf/tissue composites}

To verify that the structures in implanted regions were indeed blood vessels, immunostaining with two well established endothelial cell markers (CD31 and vWF) was used, 

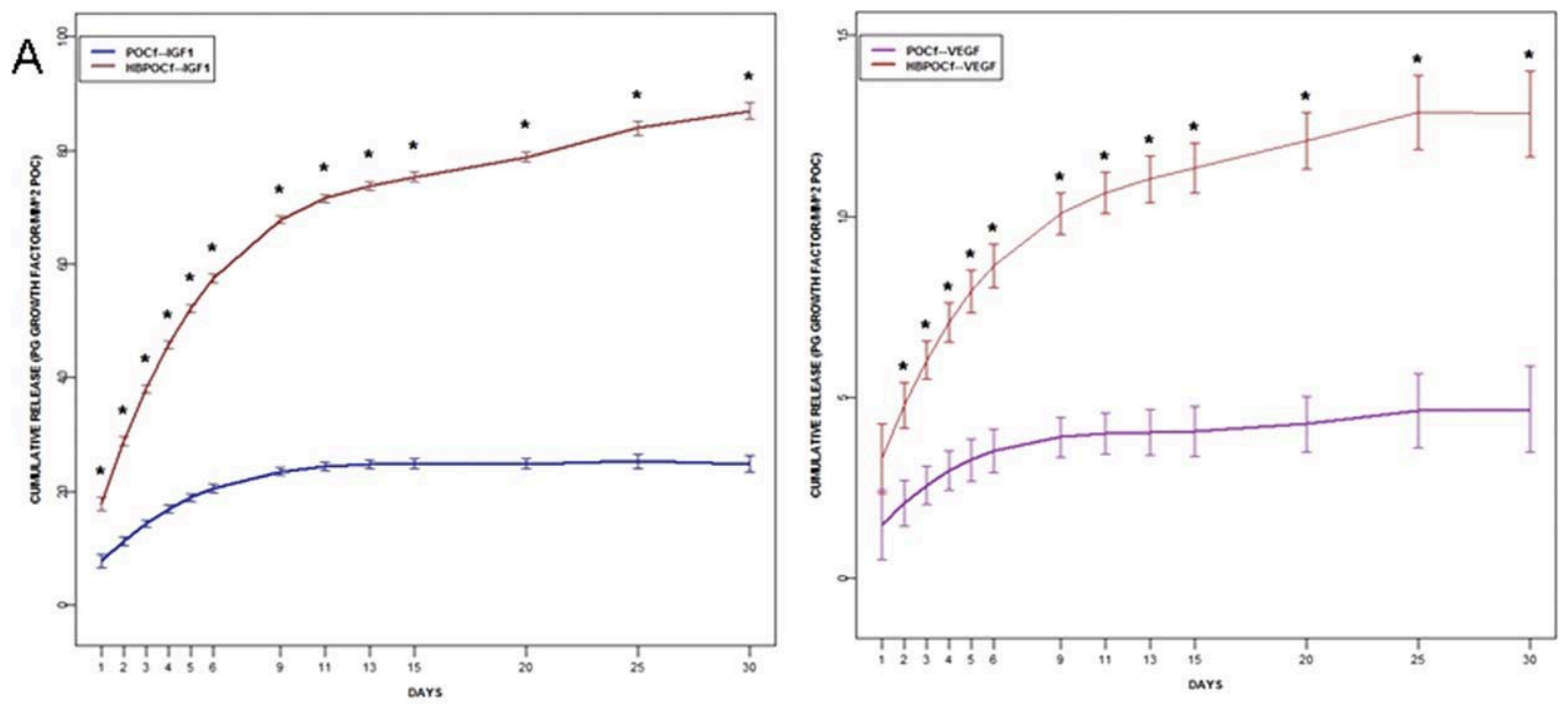

$\mathrm{B}$
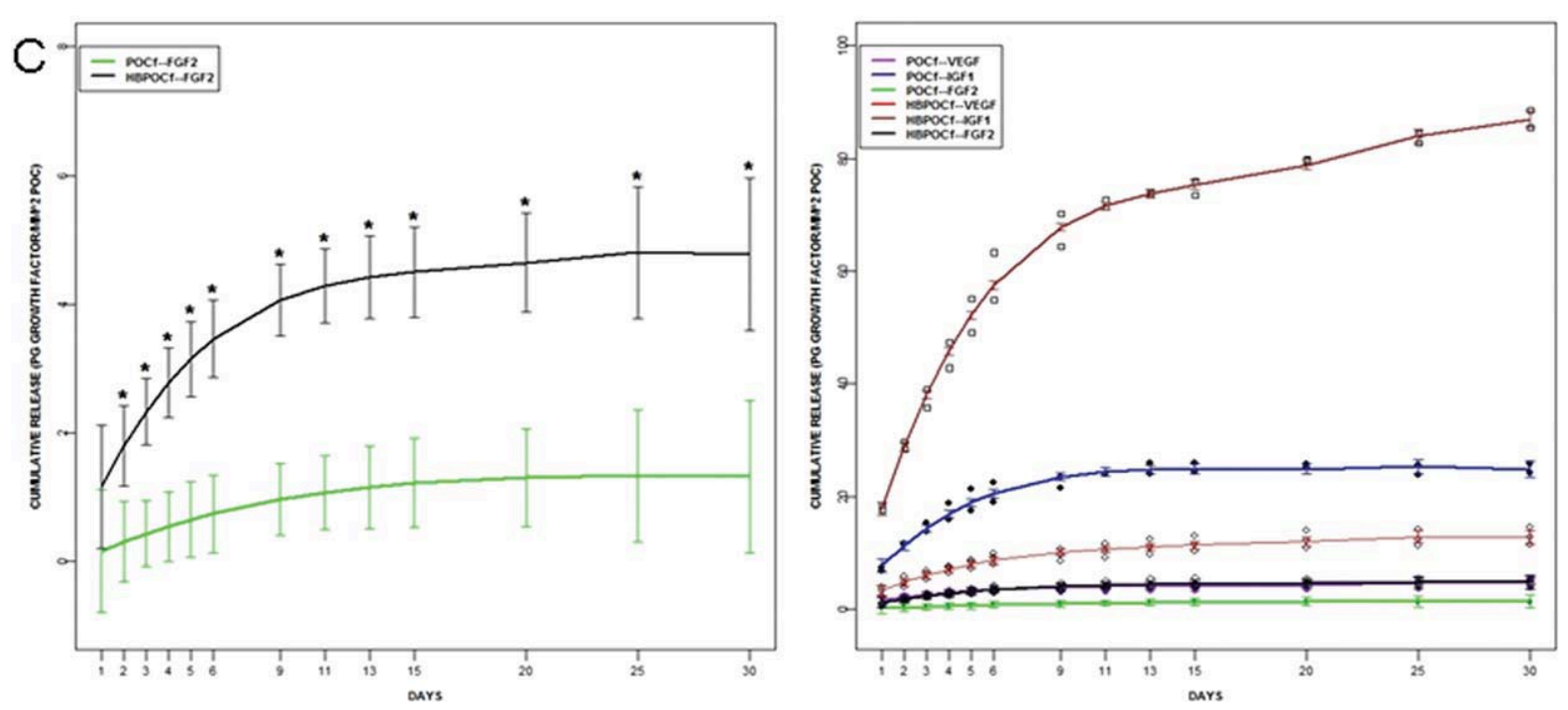

FIGURE 3. In Vitro growth factor release analyses. (A) IGF-1 constructs showed the highest levels of growth factor release, followed by VEGF constructs (B), with FGF2 constructs releasing the lowest levels (C), and (D) demonstrates HBPOCf constructs released significantly higher levels of growth factors than POCf constructs. Data shown as raw data points with fitted lines and $95 \%$ predictive intervals ( $^{*} p<0.05$ POCf-FGF2 vs. HBPOCf-FGF2 at day 2; ${ }^{*} p<0.0001$ all other comparisons). [Color figure can be viewed in the online issue, which is available at wileyonlinelibrary.com.]

as blood vessels are composed of a single layer of endothelial cells. ${ }^{21}$ Figure $6(\mathrm{~A}, \mathrm{~F})$ represents control sample tissue, while Figure $6(\mathrm{~B}, \mathrm{G})$ is POCf tissue samples stained with CD31 and vWF, respectively. Data demonstrate scant vascularization in these areas. HBPOCf implanted samples [Fig. $6(\mathrm{C}, \mathrm{H})]$ are similar in vessel representation as the control and POCf samples. POCf-VEGF containing samples are similarly stained in Figure 6(D,I) and show an increase level in vasculature in the implanted area. Finally, Figure 6(E,J) demonstrates the biggest increase in vessel density and size with positive staining for both endothelial cell markers. Immunofluorescent data suggest that vascular levels were significantly higher in HBPOCf-VEGF constructs than controls. Data were consistent in all animals and further corroborated by vessel quantification studies performed in the next subsection. The apparent yellow fluorescence found within the lumen of blood vessels is due to red blood cell autofluorescence [most apparent in Fig. 6(D)] (400× mag; scale bar $=50 \mu \mathrm{m})$.

\section{Vasculature quantification in implanted areas}

HBPOCf-VEGF implants produced a significantly enhanced vascular response in the surrounding tissue as compared with POCfs, HBPOCfs, and POCfs-VEGF. The addition of heparan conjugated VEGF resulted in approximately four times the response observed with POCf or HBPOCf alone and approximately two times the response seen with the addition of free VEGF. Specifically, mean values $(n=10-11$ implants per condition) were $41.1 \pm 4.1$ vessels $/ \mathrm{mm}^{2}$ region for the POCf group, $41.7 \pm 2.6$ vessels $/ \mathrm{mm}^{2}$ for the HBPOCf group, $76.3 \pm 9.4$ vessels $/ \mathrm{mm}^{2}$ for the POCf-VEGF group, and $167.7 \pm 15.3$ vessels $/ \mathrm{mm}^{2}$ for the HBPOCf-VEGF 

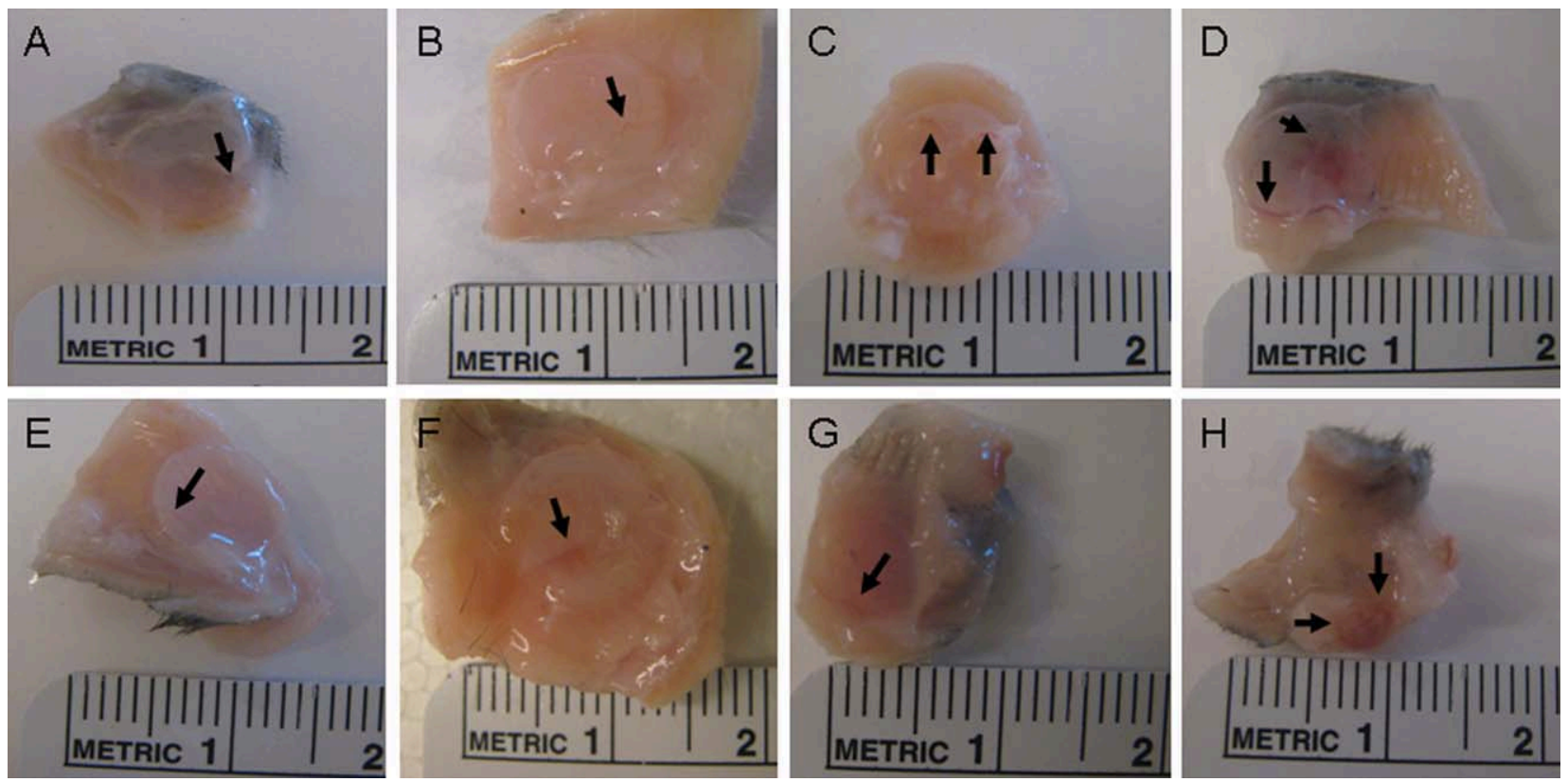

FIGURE 4. Macroscopic analyses of 4-week POCf implanted tissue specimens: $(A, E)$ depict subcutaneously implanted POCfs removed from the dorsal area of a nude rat, Note the scarcity of blood vessels, $(B, F)$ depict implanted HBPOCfs, and $(C, G)$ are similar conditions but implanted with POCfs-VEGF. The level of vascularity increases with the addition nonchemically conjugated VEGF. (D,H) shows the highest levels of localized vascularization using the HBPOCfs-VEGF subcutaneously implanted as indicated by the black arrows $(\times 400$ mag; scale bar $=50 \mu \mathrm{m})$. (A-D) and $(\mathrm{E}-\mathrm{H})$ depict two different animals, respectively. [Color figure can be viewed in the online issue, which is available at wileyonlinelibrary.com.]

group ( $n=5$ animals) (Fig. 7). Nonimplant control samples displayed $38.8 \pm 6.1$ vessels $/ \mathrm{mm}^{2}$ were similar to POCf and HBPOCf control samples. Thus, the increased vascular response conferred by addition of free VEGF is further enhanced by VEGF presented in the heparan-binding construct.

\section{DISCUSSION}

The biological processes by which an organ undergoes regeneration in vivo are influenced by numerous environmental parameters. These include cell-interface interactions along with cellular modulation by soluble and nonsoluble factors, which may be autocrine or paracrine in nature that modulate re-cellularization, cell migration, and growth. ${ }^{23,24}$ Dimensionality and mechanical stimulation also play significant roles in the formation and differentiation of cellderived tissues since the native states of regenerative processes occur in three dimensions, hence choice of a scaffold and its mechanical properties are crucial for proper organ formation for regenerative purposes. ${ }^{15,25}$ In the field of urological tissue engineering, great strides have been made over the last several years to create replacement structures
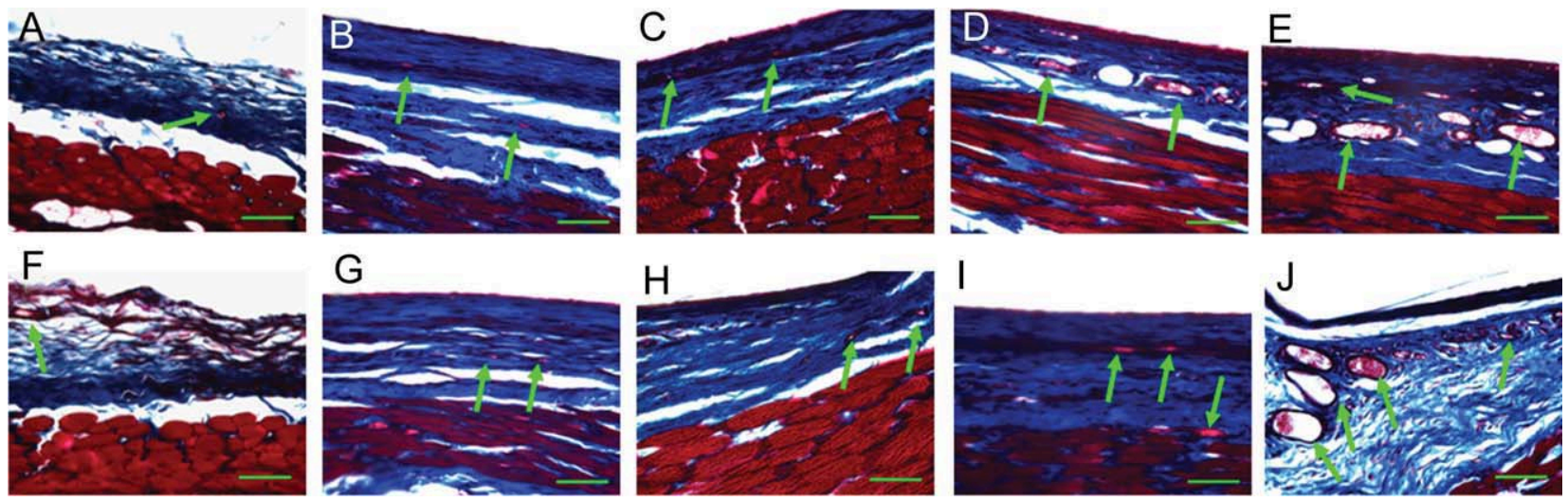

FIGURE 5. Masson's Trichrome Staining of 4-week POCf implanted tissue specimens: (A,F) are histological staining of tissue samples taken from the dorsal area of unmanipulated, control nude rats, $(B, G)$ are POCf implanted animals, while $(C, H)$ represent $H B P O C f$ implanted films. $(D, I)$ demonstrate an increase in blood vessel formation with the addition of the POCf-VEGF construct, while the greatest difference in vascularization is seen in $(E, J)$ containing the HBPOCf-VEGF constructs. Vessels are indicated by green arrows. Note the difference in vessel size from the HBPOCf-VEGF to the POCf alone ( $\times 400$ mag; scale bar $=50 \mu \mathrm{m})$. (A-E) and (F-J) depict two different animals, respectively. [Color figure can be viewed in the online issue, which is available at wileyonlinelibrary.com.] 

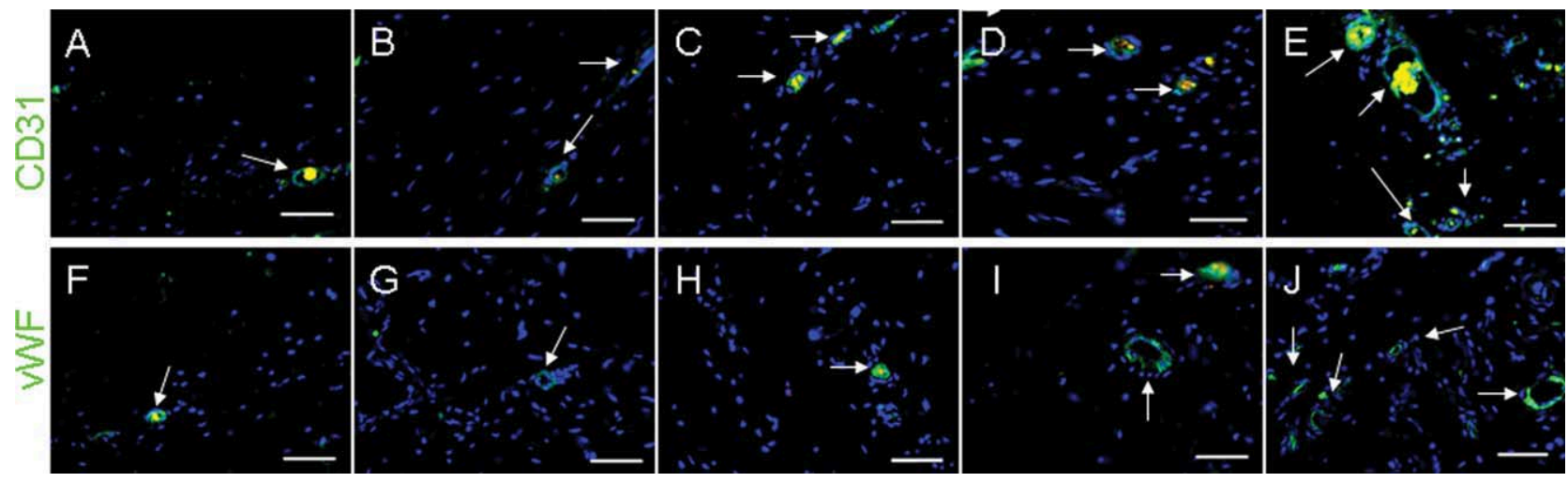

FIGURE 6. Immunofluorescent imaging of POCf/cell tissues 4-weeks post augmentation. Antibodies against known endothelial cell markers CD31 and VWF typically found in vasculature were used to identify blood vessels. (A) Represents unmanipulated control animal tissue stained with CD31/FITC antibody (green). (B) POCf implanted tissue composite stained with CD31/FITC antibody. (C) HBPOCf implanted tissue composite stained with CD31/FITC antibody. (D) POCf-VEGF implanted tissue composite stained with CD31/FITC antibody. (E) HBPOCf-VEGF implanted tissue composite stained with CD31/FITC antibody. (F) Unmanipulated control animal tissue stained with vWF/FITC (green). (G) POCf implanted tissue composite stained with vWF-FITC antibody. (H) HBPOCf implanted tissue composite stained with vWF-FITC. (I): POCf-VEGF implanted tissue composite stained with VWF-FITC antibody. (J) HBPOCf-VEGF implanted tissue composite stained with vWF-FITC antibody. White arrows indicate vasculature. All samples counter stained with DAPI (blue) to identify cell nuclei ( $\times 400$ mag; scale bar $=50 \mu \mathrm{m}$ ). [Color figure can be viewed in the online issue, which is available at wileyonlinelibrary.com.]

to be used for a variety of urological applications using cells and either synthetic or biological-based scaffolds. ${ }^{5,14,26,27}$ If one were to simplistically break down the components of the bladder, it would include the urothelium that lines that interior of the bladder allowing for a urine tight barrier; the musculature that allows for repeated contraction/expansion cycles; neural conduits; and a vascular supply. Apparent pathogenic and nonpathogenic sources of cells have been used to create the urothelial and muscle components of the bladder with varying degrees of success. ${ }^{5,14}$ Severely lacking in these models are schemes to create functional neural components and highly interconnected networks of robust vascularization. The ability to provide a foundation in which adequate vascularization encompasses all aspects of the regenerating bladder would be invaluable. This study was performed as a proof of concept to demonstrate that the chemical alteration of POC could provide a basis for directed angiogenesis in vivo.

Previous studies using MSC seeded POCfs composites demonstrated that these composites could function as surrogate bladder tissue in a partial bladder cystectomy model. ${ }^{14}$ Data from this study demonstrated that both seeded and unseeded POCfs were impermeable to urine at the onset of the study and provided this barrier until native urothelium invaded and grew into the graft. ${ }^{14}$ Although the study provided convincing evidence that bladder regeneration took place using MSC/POC scaffolds, we postulated that regeneration may be greatly enhanced with the localized deposition of growth factors known to promote angiogenesis and smooth muscle development. ${ }^{12}$ This was based on the observation that areas of regeneration were not as highly vascularized as neighboring native tissue. Second, bladder regeneration strategies often employ the use of omentum to cover a newly transplanted bladder to provide a potential source of vascularization to the newly implanted bladder tissue. ${ }^{4}$ Within this scenario, the omentum is left in place over the lifetime of the graft. Although this may act as a means for vascular supply, the long-term effects of omentum intermingling with bladder tissue have not been thoroughly evaluated. The omentum produces a wide variety of hormones and growth factors that may be detrimental to the regenerative process including proinflammatory cytokines and chemokines. ${ }^{28}$ To avoid the future use of omentum, POC was modified to release growth factors that could possibly aid in the vascularization process. Initial in vitro testing and subsequent growth factor release data from this study demonstrated unique differences in the release profiles of the three growth factors examined. The three POCf constructs provided evidence of sustained growth factor release over time in which the POCf-IGF-1 construct showed the highest levels of release $\left(25.0 \pm 1.7 \mathrm{pg} \mathrm{GF} / \mathrm{mm}^{2} \mathrm{POC}\right)$. This was significantly greater than POCf-VEGF $(4.7 \pm 0.9 \mathrm{pg}$ $\left.\mathrm{GF} / \mathrm{mm}^{2} \mathrm{POC}\right)$ and also greater than POCf-FGF2 (1.3 \pm 0.1

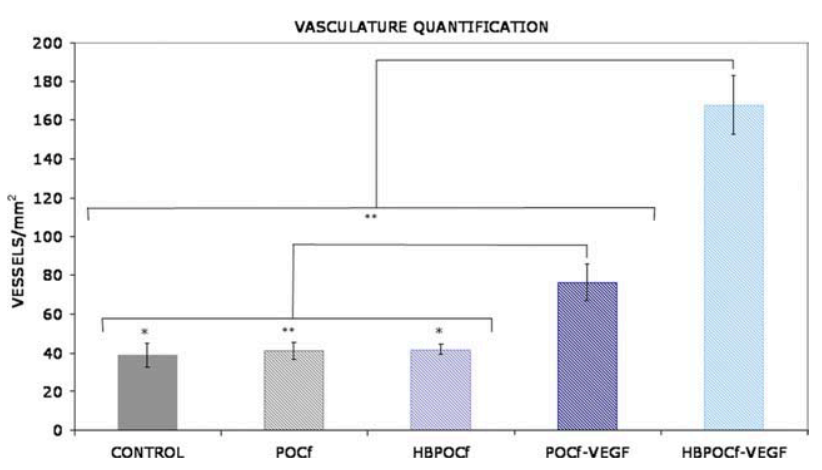

FIGURE 7. In vivo vasculature quantification analyses. HBPOCf-VEGF implants produced a significantly greater vascular response than POCf, HBPOCf, or POCf-VEGF implants. Data shown as number of vessels per $\mathrm{mm}^{2}$ represent mean $\pm 95 \%$ confidence intervals for 12 fields surveyed per implant ( $n=10-11$ implants per condition) ( $^{*} p<$ $0.01 ;{ }^{*} p<0.0001$ ). [Color figure can be viewed in the online issue, which is available at wileyonlinelibrary.com.] 
pg GF/ $\mathrm{mm}^{2}$ POC) at all time points at 30 days. At 30 days, release amounts were $87.0 \pm 3.1\left(\mathrm{pg} \mathrm{GF} / \mathrm{mm}^{2}\right.$ POC) HBPOCf-IGF-1 versus $12.9 \pm 1.8\left(\mathrm{pg} \mathrm{GF} / \mathrm{mm}^{2}\right.$ POC) HBPOCf-VEGF versus $4.8 \pm 0.6\left(\mathrm{pg} \mathrm{GF} / \mathrm{mm}^{2}\right.$ POC) HBPOCfIGF-1. The bulk release of growth factors with these POCf constructs occurred by days 5-6 where $\sim 75 \%$ of VEGF and IGF-1 were released and $50 \%$ of FGF2. In stark contrast, growth factors bound to HBPOCfs required $\sim 9$ days of incubation before $75 \%$ of the growth factor was released with a burst release of $\sim 50 \%$ of the growth factors released by day 4 . Since heparan sulfate is a highly sulfated glycosaminoglycan, it may have provided a protective effect to bound growth factors that allowed for their extended in vitro release as many of these growth factors have a very short half-life. ${ }^{29,30}$

The release profiles of these molecules appear quite disparate and may be dependent upon several factors including the number of heparan binding motifs contained within each molecule as well as their respective molecular masses. In the aforementioned data, IGF-1 consistently demonstrated the highest level of release in both types of POCf constructs. The predicted molecular mass for IGF-1 is $7.5 \mathrm{kDa}$, while VEGF and FGF2 are 19.2 and $17.4 \mathrm{kDa}$, respectively. ${ }^{31-33}$ The reduced molecular mass of IGF-1 as compared with VEGF and FGF2 may allow for higher numbers of IGF-1 molecules to occupy any given space hence the higher level of release. The study of these particular three growth factors in vitro was to demonstrate that the HBPOCfs possessed the capability to bind and release three different classes of proangiogenic growth factors. Secondary factors that may have affected the differences in release profiles can be attributed to the surface charge of the differing POCfs as well as the shape and texturing of the POCfs as was demonstrated by AFM data.

Materials that are used to act as scaffolds for tissue engineering purposes come in many different embodiments. One parameter of synthetically produced scaffolds that has been recently shown to effect cellular growth is the surface topography. ${ }^{34}$ Different methods have been used to specifically create surfaces with texture, roughness, or patterns in order for cells to receive cues to perform particular functions. ${ }^{35,36}$ It has been further demonstrated that rougher surfaces tend to foster cell growth and enhance adhesions between cells and material, particularly in a urological setting. ${ }^{37}$ This may be in part due to the notion that biomaterial surfaces have comparable dimensional qualities to proteins that inherently stud cell surfaces, for example. This is in contrast to material surfaces that are less rough or lacking this aspect completely. AFM data presented within this study demonstrate that POCf and HBPOCf displayed similar average roughness values, while HBPOCf-VEGF constructs contained a higher average roughness value and broader surface distance with the presence of numerous hills and valleys motifs as demonstrated in Figure 2(C). In vivo vessel quantification data of implanted scaffolds demonstrate that the new vessel growth occurred at its highest levels in the presence of the HBPOCf-VEGF that also expressed the highest degree of surface roughness. Data indicate that HBPOCf-
VEGF displayed an approximate fourfold and twofold increase in vessel number compared with POCf and POCfVEGF, respectively. We speculate that the combination of heparan bound growth factor and roughness allowed for superior vascular growth as compared with the more "slick" surfaces found in the POCf and POCf-VEGF constructs. Cells from the local subcutaneous environment may have been recruited by growth factor signals and were able to encroach and populate the HBPOCf-VEGF construct at multiple depths/heights, forming close knit three-dimensional cellular niches that may have been conducive to vascular growth. However, one must take into account scalability with regard to roughness. Objects that appear to be smooth in appearance at one particular scale may indeed be textured at a smaller scale. An examination into the degree of surface texture at various scales needs to be further investigated as to eventually synthesize a scaffold with a textured surface that is highly conducive to cell growth and development.

The distribution of newly generated blood vessels in HBPOCf-VEGF constructs appeared to be situated in certain areas of the graft. Figure 4(C,F) displays areas of localized angiogenesis that are demarcated by the black arrows. It is evident in Figure 4(C) that the blood vessels are concentrated in a specific region of the HBPOCf-VEGF as the outline of the circumference of the HBPOCf-VEGF can be visualized. This may be due to several reasons including a localized concentration of VEGF as well as the duration in which the graft was left in place. The chemistry involved in the conjugation of the PEG spacer arm to the POCf would indicate a 1:1 addition of PEG to free carboxylic acid groups found on the POCfs. Although every effort was used to evenly distribute the GFs onto the HBPOCf, alternative methods may be employed in the future to ensure a better so that optimal results may be achieved when films are placed in an in vivo tissue regenerative environment. Finally, the in vivo environment and the length of time in which the grafts remained also play a key role in the level of angiogenesis. Vessel formation may have become more homogenous in distribution over time lending to a more robust level of regeneration. Aside from these points, the data obtained from this study clearly demonstrate increased angiogenesis in HBPOCfs-VEGF as compared with controls at the gross, microscopic, and quantitative levels.

\section{REFERENCES}

1. Bankhead RW, Kropp BP, Cheng EY. Evaluation and treatment of children with neurogenic bladders. J Child Neurol 2000;15: 141-149.

2. Brown AL, Brook-Allred TT, Waddell JE, White J, Werkmeister JA, Ramshaw JA, Bagli DJ, Woodhouse KA. Bladder acellular matrix as a substrate for studying in vitro bladder smooth muscle-urothelial cell interactions. Biomaterials 2005;26:529-543.

3. Loai Y, Yeger H, Coz C, Antoon R, Islam SS, Moore K, Moore K, Farhat WA. Bladder tissue engineering: Tissue regeneration and neovascularization of HA-VEGF-incorporated bladder acellular constructs in mouse and porcine animal models. J Biomed Mater Res A 2010;15:1205-1215.

4. Mondalek FG, Ashley RA, Roth CC, Kibar Y, Shakir N, Ihnat MA, Fung KM, Grady BP, Kropp BP, Lin HK. Enhanced angiogenesis of modified porcine small intestinal submucosa with hyaluronic 
acid-poly(lactide-co-glycolide) nanoparticles: From fabrication to preclinical validation. J Biomed Mater Res A 2010;94:712-719.

5. Atala A, Bauer SB, Soker S, Yoo JJ, Retik AB. Tissue-engineered autologous bladders for patients needing cystoplasty. Lancet 2006;367:1241-1246.

6. Rajangam K, Behanna HA, Hui MJ, Han X, Hulvat JF, Lomasney JW, Stupp SI. Heparin binding nanostructures to promote growth of blood vessels. Nano Lett 2006;6:2086-2090.

7. Cardin AD, Weintraub HJ. Molecular modeling of protein-glycosaminoglycan interactions. Arteriosclerosis 1989;9:21-32.

8. Fromm JR, Hileman RE, Caldwell EE, Weiler JM, Linhardt RJ. Pattern and spacing of basic amino acids in heparin binding sites. Arch Biochem Biophys 1997;343:92-100.

9. Losi P, Briganti E, Magera A, Spiller D, Ristori C, Battolla B, Balderi M, Kull S, Balbarini A, Di Stefano R, Soldani G. Tissue response to poly(ether)urethane-polydimethylsiloxane-fibrin composite scaffolds for controlled delivery of pro-angiogenic growth factors. Biomaterials 2010: 31:5336-5344.

10. Davies N, Dobner S, Bezuidenhout D, Schmidt C, Beck M, Zisch $\mathrm{AH}$, Zilla $\mathrm{P}$. The dosage dependence of VEGF stimulation on scaffold neovascularisation. Biomaterials 2008;29:3531-3538.

11. Dahms SE, Piechota HJ, Dahiya R, Lue TF, Tanagho EA. Composition and biomechanical properties of the bladder acellular matrix graft: Comparative analysis in rat, pig and human. Br J Urol 1998; 82:411-419.

12. Yang J, Motlagh D, Webb AR, Ameer GA. Novel biphasic elastomeric scaffold for small-diameter blood vessel tissue engineering. Tissue Eng 2005;11:1876-1886.

13. Yang J, Webb AR, Pickerill SJ, Hageman G, Ameer GA. Synthesis and evaluation of poly(diol citrate) biodegradable elastomers. Biomaterials 2006;27:1889-1898.

14. Sharma AK, Hota PV, Matoka DJ, Fuller NJ, Jandali D, Thaker $\mathrm{H}_{\text {, }}$ Ameer GA, Cheng EY. Urinary bladder smooth muscle regeneration utilizing bone marrow derived mesenchymal stem cell seeded elastomeric poly(1,8-octanediol-co-citrate) based thin films. Biomaterials 2010;31:6207-6217.

15. Heise RL, Ivanova J, Parekh A, Sacks MS. Generating elastin-rich small intestinal submucosa-based smooth muscle constructs utilizing exogenous growth factors and cyclic mechanical stimulation. Tissue Eng Part A 2009;15:3951-3960.

16. Paredes JI, Villar-Rodil S, Solís-Fernández $P$, Martínez-Alonso $A$ Tascón JM. Atomic force and scanning tunneling microscopy imaging of graphene nanosheets derived from graphite oxide. Langmuir 2009;25:5957-5968.

17. Chow LW, Bitton R, Webber MJ, Carvajal D, Shull KR, Sharma AK, Stupp SI. A bioactive self-assembled membrane to promote angiogenesis. Biomaterials 2011;32:1574-1582.

18. Brady G, O'Regan E, Miller I, Ogungbowale A, Kapas S, Crean SJ. Serum levels of insulin-like growth factors (IGFs) and their bind ing proteins (IGFBPs), $-1,-2,-3$, in oral cancer. Int J Oral Maxillofac Surg 2007;36:259-262.

19. Graeven U, Andre N, Achilles E, Zornig C, Schmiegel W. Serum levels of vascular endothelial growth factor and basic fibroblast growth factor in patients with soft-tissue sarcoma. J Cancer Res Clin Oncol 1999;125:577-581.

20. Wang Y, Rudym DD, Walsh A, Abrahamsen L, Kim HJ, Kim HS, Kim HS, Kirker-Head C, Kaplan DL. In vivo degradation of threedimensional silk fibroin scaffolds. Biomaterials 2008;29: 3415-3428.

21. Pusztaszeri MP, Seelentag W, Bosman FT. Immunohistochemical expression of endothelial markers CD31, CD34, von Willebrand factor, and Fli-1 in normal human tissues. J Histochem Cytochem 2006;54:385-395.

22. Mazzitelli C, Ferrari M, Toledano M, Osorio E, Monticelli F, Osorio R. Surface roughness analysis of fiber post conditioning processes. J Dent Res 2008;87:186-190.

23. Morrell NT, Leucht $P$, Zhao L, Kim JB, ten Berge D, Ponnusamy $K$, Carre $A L$, Dudek $H$, Zachlederova $M$, McElhaney $M$, Brunton $S$, Gunzner J, Callow M, Polakis P, Costa M, Zhang XM, Helms JA, Nusse R. Liposomal packaging generates Wnt protein with in vivo biological activity. PLoS One 2008;A3:e2930.

24. Adon AM, Zeng $X$, Harrison MK, Sannem S, Kiyokawa $H$, Kaldis $\mathrm{P}$, Saavedra HI. Cdk2 and Cdk4 regulate the centrosome cycle and are critical mediators of centrosome amplification in p53-null cells. Mol Cell Biol 2010;30:694-710.

25. Chowdhury F, Na S, Li D, Poh YC, Tanaka TS, Wang F, Wang N. Material properties of the cell dictate stress-induced spreading and differentiation in embryonic stem cells. Nat Mater 2010;9: 82-88.

26. Cannon TW, Sweeney DD, Conway DA, Kamo I, Yoshimura N, Sacks M, Chancellor MB. A tissue-engineered suburethral sling in an animal model of stress urinary incontinence. BJU Int 2005;96: 664-669.

27. Sharma AK, Bury MI, Marks AJ, Fuller NJ, Meisner JW, Tapaskar N, Halliday LC, Matoka DJ, Cheng EY. A non-human primate model for urinary bladder regeneration utilizing autologous sources of bone marrow derived mesenchymal stem cells. Stem Cells 2011;29:241-250.

28. Pérez-Pérez R, Ortega-Delgado FJ, García-Santos E, López JA, Camafeita E, Ricart W, Fernández-Real JM, Peral B. Differential proteomics of omental and subcutaneous adipose tissue reflects their unalike biochemical and metabolic properties. J Proteome Res 2009;8:1682.

29. Zern BJ, Chu H, Wang Y. Control growth factor release using a self-assembled [polycation:heparin] complex. PLoS One 2010;5: e11017.

30. George ML, Eccles SA, Tutton MG, Abulafi AM, Swift RI. Correlation of plasma and serum vascular endothelial growth factor levels with platelet count in colorectal cancer: Clinical evidence of platelet scavenging? Clin Cancer Res 2000;6:3147-3152.

31. Mohammadi M, Olsen SK, Goetz R. A protein canyon in the FGFFGF receptor dimer selects from an à la carte menu of heparan sulfate motifs. Curr Opin Struct Biol 2005;15:506-516.

32. Guvakova MA. Insulin-like growth factors control cell migration in health and disease. Int J Biochem Cell Biol 2007;39:890-909.

33. Weis SM, Cheresh DA. Pathophysiological consequences of VEGF-induced vascular permeability. Nature 2005;437:497-504.

34. Jandt KD, Finke M, Cacciafesta P. Aspects of the physical chemistry of polymers, biomaterials and mineralised tissues investigated with atomic force microscopy (AFM). Colloids Surf B Biointerfaces 2000;19:301-314.

35. Kim EJ, Boehm CA, Mata A, Fleischman AJ, Muschler GF, Roy S. Post microtextures accelerate cell proliferation and osteogenesis. Acta Biomater 2010;6:160-169.

36. Biggs MJ, Richards RG, McFarlane S, Wilkinson CD, Oreffo RO, Dalby MJ. Adhesion formation of primary human osteoblasts and the functional response of mesenchymal stem cells to $330 \mathrm{~nm}$ deep microgrooves. J R Soc Interface 2008;5:1231-1242.

37. Chun YW, Khang D, Haberstroh KM, Webster TJ. The role of polymer nanosurface roughness and submicron pores in improving bladder urothelial cell density and inhibiting calcium oxalate stone formation. Nanotechnology 2009;20:085104. 